\title{
HALO CONTAINMENT THROUGH A LANDAU DAMPING MECHANISM
}

\author{
V. Variale, INFN-Bari via Amendola 173, 70126-Bari, Italy
}

\begin{abstract}
The rms beam envelope oscillations, risen from the mismatching of high intensity beams propagating along periodic focusing cells, can induce a halo formation. In some case, a very fast oscillation damping, due in all probability to a Landau mechanism of stabilization, can occur minimizing the halo extent generated.

In this paper the possibility of using this fast damping to contain the halo formation on the beam during the transportation along periodic focusing cells will be explored and discussed.
\end{abstract}

\section{INTRODUCTION}

High intensity ion beams are required for many interesting technological and scientific applications [1]. Among them, there is the possibility of using a high intensity ion beam for the energy amplifier and the nuclear wave transmutation [2].

The TRASCO project it is an Italian feasibility study aiming to design the main components of an accelerator driven system (ADS) for nuclear waste transmutation [3]. In the framework of this project, simulations to study problems related to the high intensity beam transportation through periodic FODO cells, in mismatched conditions, are been carried out. Very fast damping of beam envelope oscillation amplitudes has been observed in those simulations [4,5]. Since the cause of the halo formation seems due, mainly, to the mismatching [6], the study of this effect could be interesting for understanding how to contain the halo extent. From the simulation results, it seems that the fast damping observed could be ascribed to a Landau damping mechanism [5]

In this paper, in order to point out in which conditions the fast damping observed in [5], mainly for the odd mismatched beams, occurs also for the even mismatched ones, several simulations have been carried out. Furthermore, tentative parameters to maximize the even fast damping strength will be investigated.

\section{SIMULATIONS}

The multiparticle code PARMT [7], used for the simulations, is a Monte Carlo program that can transport an ion beam through a system of optic elements by using the matrix method. Being simulated a very high number of particles; PARMT uses a 'Fast Poisson Solver' (FPS) technique to compute the space charge electric field of the ion beam. The fast damping effect of the rms envelope oscillations, for a continuous beam transported through FODO cells, in mismatched conditions, has been already shown in ref. [5]. As reported in [5], this effect can be ascribed to a Landau Damping (LD) mechanism of stabilization. In fact, the fast damping occurred only when the LD conditions were satisfied, that is when the envelope oscillations frequencies, due to mismatching, were covered by a large beam betatron frequency distribution. The damping of the coherent oscillation, the envelope modes, can be explained by an energy transfer towards the ensemble of incoherent oscillators, the beam particles. In other words, the energy involved in the coherent oscillation process is dissipated towards the very high number of degrees of freedom of the system (the beam). Furthermore, a parametric resonant transfer of the energy can occur only if the half frequency of the envelope modes is close to the particle betatron frequency distribution. In this case the energy coming from the coherent oscillation is shared, practically, from all the beam particles. On the other hand, if only few particles have betatron frequencies close to the coherent oscillation, the same energy will be transferred only to this small number of particles leading to a halo formation. The further simulations carried out in this paper will start from a case already shown in ref. [5]. The input beam parameter for that case was: FODO cells with a cell of 80 $\mathrm{cm}$; rms transverse emittances $\varepsilon_{\mathrm{x}}=\varepsilon_{\mathrm{y}}=0.25 \mathrm{~mm} \mathrm{x} \mathrm{mr}$; initial particle distribution of Gaussian type; beam current $\mathrm{I}=95 \mathrm{~mA}$ with a relativistic factor $\beta=0.145$ and single particle phase advance $\sigma_{0}=60.7^{\circ}$. Furthermore a mismatch of $10 \%$ of the beam size was added. The related simulation results has been again presented in fig. 1 for convenience. From that figure we can observe that the fast damping effect occurs only for the odd mismatched case and not the even one. This is because, as argued in ref [5], the even mode is on the edge of betatron frequency distribution, then does not satisfy the LD conditions. In order to include also the even mode in the fast damping conditions, let's try further simulations with some input beam parameters, as $\sigma_{0}$ and $\beta$, changed.

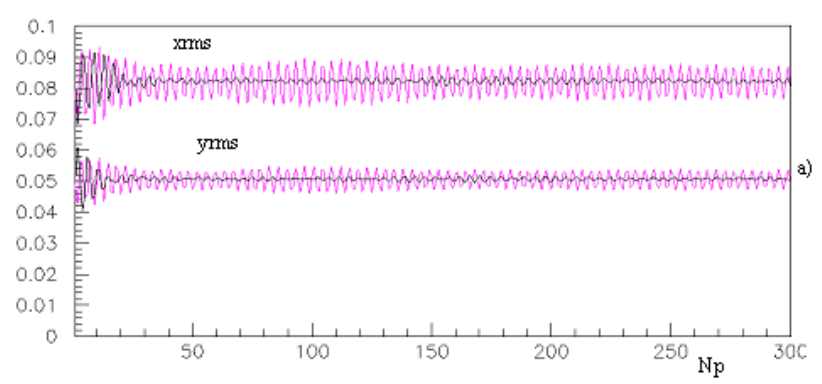




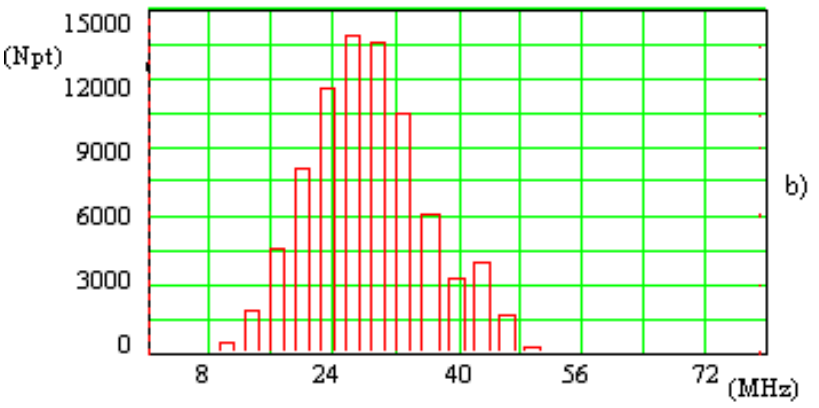

Fig.1. a) The rms envelope oscillations with initial Gaussian distribution for $\sigma / \sigma_{0}=0.55, \mathrm{~Np}$ is the periodic cell number. The black line refers to the odd mode and the magenta to the even one; b) beam betatron frequency distribution. The odd mode half frequency, $\omega^{\circ}$, is $38 \mathrm{Mhz}$ and the even one, $\omega^{\mathrm{e}}$, is $45 \mathrm{Mhz}$.

As first test we try to change the single particle phase advance $\sigma_{0}$ from $60^{\circ}$ to $75^{\circ}$ (less focusing). From the related simulation results, practically, no difference can be observed comparing them with the results shown in [5] and presented again in fig.1. This is because the increase of $\sigma_{0}$ produces also an increase of $\sigma$.
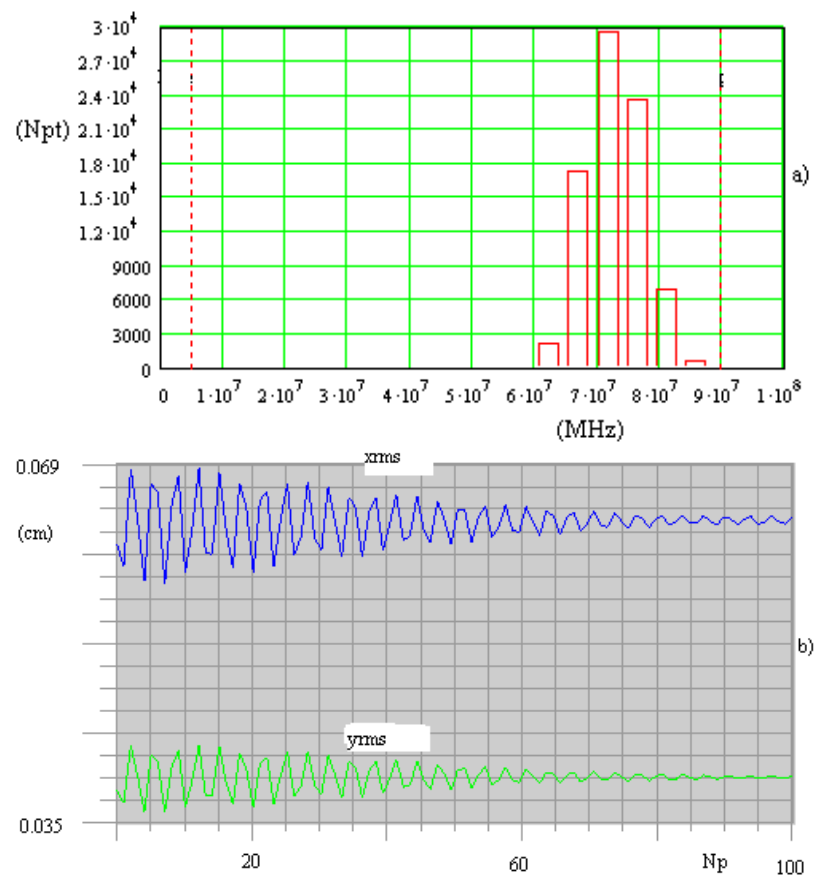

Fig. 2. a) Frequency betatron distribution for $\beta=0.22$ and $\mathrm{s} / \mathrm{s} 0=0.82$, the half frequency mode is about $79 \mathrm{MHz} ; \mathrm{b}$ ) related even mismatched beam rms envelope oscillations.

In fact, the beam matching with the FODO cell for a higher $\sigma_{0}$ is obtained for a larger beam size then a lower current density that gives also a higher $\sigma$. Furthermore, The phase advance $\sigma$ gives also a higher the central betatron frequency $\omega$. In conclusion, we have an increase not only of the even mode phase advance $\Phi_{\mathrm{e}}=\left(\sigma_{0}{ }^{2}+\sigma^{2}\right)^{1 / 2}$ [8], and then of $\omega_{\mathrm{e}}$, but also of $\sigma$. The relative frequency positions, $\omega$ and $\omega_{\mathrm{e}}$, in the spectrum, then, practically do not change. On the other hand, a change of the beam relativistic factor $\beta$, gives a more efficient changing of the relative frequency positions between the betatron spectrum $\Delta \omega_{\beta}$, centred in $\omega$, and the even mode frequency $\omega_{\mathrm{e}}$. The simulation results shown in fig. 2 presents the case with $\beta=0.22(E k=23 \mathrm{MeV})$. It produces a space charge parameter $v$, defined as $\sigma / \sigma_{0}$, of about 0.82 . It can be noticed that in fig. 2b) the fast damping occurs. Furthermore, it must be observed that the results of fig. $2 b)$ are practically the same of those shown in ref. [5] for the case in which we had a beam current $\mathrm{I}=24 \mathrm{~mA}$ and a $\beta=0.145(\mathrm{Ek}=10 \mathrm{MeV})$. In that case we had practically the same space charge parameters $v$. In fact, the relative position in the frequency spectrum between rms envelope oscillation frequency and the betatron frequency distribution, once fixed $\sigma_{0}$, depends essentially by $v$ (see fig. 3). For this reason we try further simulations leaving the same input beam parameters of above but with different $\beta$ are considered.

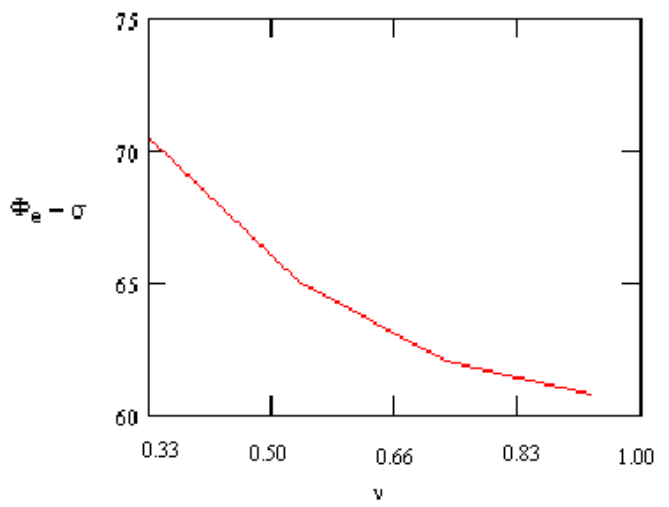

Fig. 3. Difference between the even envelope and space charge phase advance as a function of $v$.

From fig. 3, it can be seen that the $\Phi_{\mathrm{e}}-\sigma$ decreases with increasing $v$. Then the frequencies $\omega_{\mathrm{e}}$ and $\omega$ will be closer at higher value of $v$. However, a higher value of $v$ means a lower space charge force in the beam and then a narrow betatron frequency distribution. On the other side, the frequencies $\omega_{\mathrm{e}}$ and $\omega$ will be farer at lower values of $v$ but also the betatron frequency distribution will be larger being the space charge force in the beam higher. Then the LD condition will be satisfied more likely for intermediate values of $v$. In order to find out in which conditions the fastest damping effect is obtained, many simulations for different values of $v$ have been carried out.

Some of these simulation results are shown in fig. 4 where even rms envelope oscillations for the cases: $v=0.86, v=0.79, v=0.47$ are presented.

The fastest damping conditions are been found for the case $v=0.79$. However, it must be noticed that for values of $v$ close to 0.79 the simulation results change very smoothly. Furthermore, it must be observed that for lower 
values of $v$ the space charge force becomes stronger ad then the halo formation could be very important.
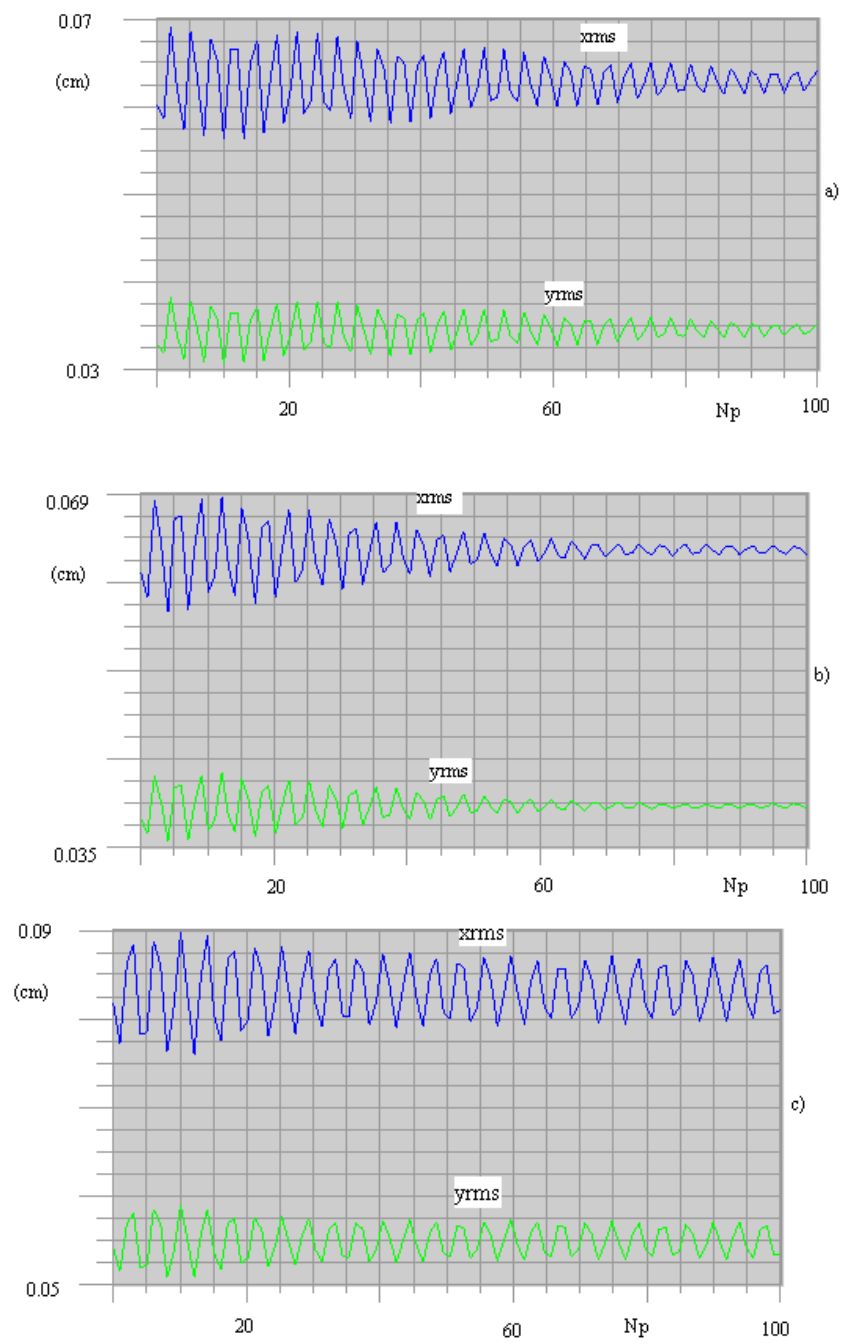

Fig. 4. The even rms oscillations for the cases: a) $\nu=0.86$, corresponding to $\beta=0.24$; b) $\nu=0.79, \beta=0.21$; c) $\mathrm{v}=0.47, \beta=0.14$.

In the case of fig. 4c), there is a high space charge force and, furthermore, only a slight damping effect can be observed. This is because the half even frequency position is close to the edge of the betatron spectrum, but not inside. In this case, a slight process of halo formation can be expected. In fact, the simulation results of fig. 5 show some particles that has left the beam core and start to form a kind of cloud around the beam. The halo extent should be larger for a higher space charge involved. The case shown in fig.6, refers to a value of $v=0.34$ and there a slightly larger halo extent can be observed.

\section{CONCLUSION}

The simulation results, presented for different $v$ values parameters, show that the fastest even damping effect can
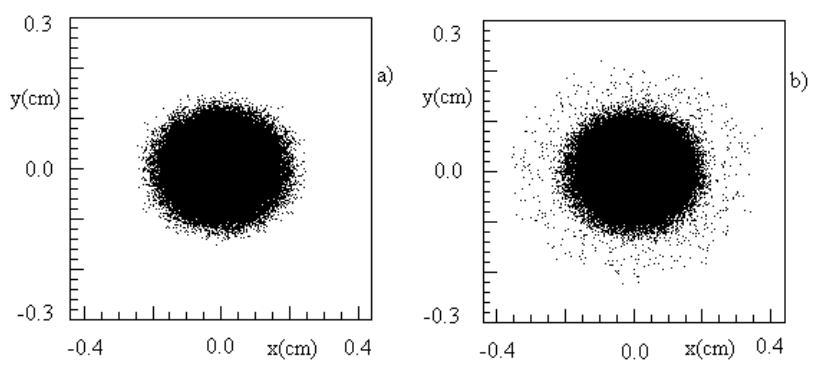

Fig. 6. Beam spots for $\nu=0.47$, corresponding to $\beta=0.14$ : a) initial condition; b) after $100 \mathrm{~Np}$.

be obtained for $v$ values of about 0.79 . However, for higher values of $\mathrm{n}$ the space charge force felt by the beam particles is lower, and then, the halo formation is of low importance. On the other hand, for $v>0.47$, the damping becomes very slight and although the low statistic, only 80000 macro particle used, the halo formation can be evident. Further lower values of $v$ produce halo with larger extent.
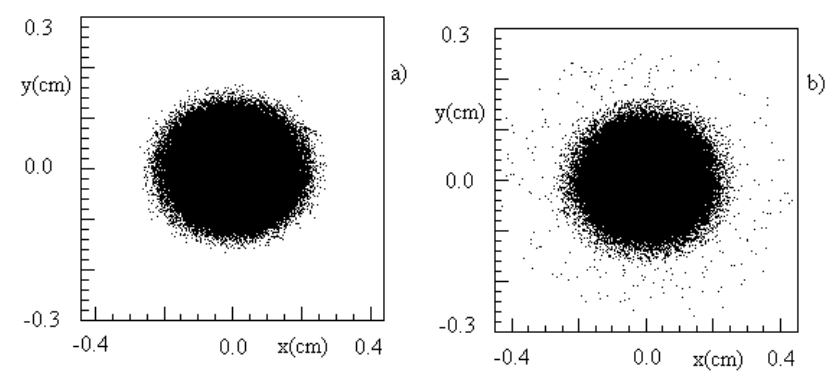

Fig. 7: Beam spots for $\nu=0.34$, corresponding to $\beta=0.11$ : a) initial condition; b) after $100 \mathrm{~Np}$.

\section{REFERENCES}

[1] See for example:' Space Charge Dominated beams and Applications of High Brightness Beams', edited by S. Y. Lee, (1995), Bloominghton, In.

[2] R. A. Jameson, G.P. Lawrence and S.O. Schriber, EPAC'92, Berlin, June 1992.

[3] M. Napolitano et al. 'Status of the high current proton accelerator for the TRASCO program', INFN/TC$00 / 23$.

[4] V. Variale," Simulation Comparison on Halo Formation between Particle Core Model and a Multiparticle code", Il Nuovo Cimento- Vol.112A, N.12 (1999)1571-1582.

[5] V.Variale, "Fast Damping in High Intensity Beam Transportation", submitted to Phys. Rev. ST-AB.

[6] M. Reiser, Proc. of the IEEE 1991 PAC 1991, edited by L. Lizama and J. Chew (IEEE, New York, 1991), p. 2497.

[7] J. Strukmeier, GSI-ESR-03 report.

[8] J. Struckmeier and M. Reiser, Part. Accel. 14, 227 (1984). 